\title{
Nanosecond laser pulse induced vertical movement of thin gold films on silicon determined by a modified Michelson interferometer
}

\author{
F. Kneier · T. Geldhauser * E. Scheer · P. Leiderer • \\ J. Boneberg
}

\begin{abstract}
The vertical movement of a $40 \mathrm{~nm}$ thin Au film on a silicon substrate during intense nanosecond (ns) laser irradiation is determined on the nm vertical and ns time scales using an optimized Michelson interferometer. The balanced setup with two detectors uses the inverse interference signal and accounts for transient reflectivity changes during irradiation. We show that a change in phase shift upon reflection must be taken into account to gain quantitative results. Three distinct fluence regimes can be distinguished, characterized by transient reflectivity behavior, dewetting processes and film detachment. Maximum displacement velocities are determined to be $0.6 \mathrm{~m} / \mathrm{s}$ and $1.9 \mathrm{~m} / \mathrm{s}$ below and above the melting threshold of the metal, respectively. Flight velocities of detaching liquid films are found to be between 30 and $70 \mathrm{~m} / \mathrm{s}$ for many nanoseconds.
\end{abstract}

\section{Introduction}

The interaction of intense nanosecond (ns)-laser pulses with surfaces comprises the immediate heating of a layer with a thickness of the wavelength-dependent absorption length. During the pulse and its active energy deposition into the material, the heat diffuses into the substrate; thus, a layer equal to the heat diffusion length becomes affected. The typical thickness of this layer ranges from below $100 \mathrm{~nm}$ on low-conductivity substrates, such as glass, to above

F. Kneier (区S) · T. Geldhauser - E. Scheer · P. Leiderer

J. Boneberg

University of Konstanz, Universitätsstrasse 10,78457 Konstanz

Germany

e-mail: fabian.kneier@uni-konstanz.de

Fax: +49-7531-883791
$1300 \mathrm{~nm}$ on highly conducting materials, such as e.g. silicon. This heating of the layer leads to a thermal expansion and thus a movement of the surface. Additional surface movement might be induced by melting. This movement in the nanometer regime is, in most applications, not specially considered.

Nevertheless, with the increasing importance of nanomechanics, thermally induced dynamics of surfaces has moved into the focus of interest. Methods for studying these surface movements include scanning probe techniques, e.g. scanning tunneling microscopy [1-4], X-ray diffraction [5-7] as well as interferometric measurements [8-10]. Furthermore, indirect methods of detection might be used such as the detachment of adsorbed particles [11].

Moreover, for the description of nanomechanical mechanisms, detailed information on the thermal expansion is required. Recently, the creation of nanostructures on thin metal films by means of intense ns-laser interference lithography and the observation of hollow structures have led to the necessity of knowing the expansion dynamics on a ns time scale in order to be able to explain structure formation [12]. Thus, the determination of the ns surface vertical movement on a quantitative level is of fundamental importance in many nanomechanical applications and especially in its role for thin-film nanostructure formation.

In the following we present here results on the expansion dynamics of a thin $(40 \mathrm{~nm})$ film of gold on a $675 \mu \mathrm{m}$ thick Si substrate with (111) orientation on the nanosecond time scale.

\section{Experimental setup}

A frequency-doubled Nd:YAG laser $(\lambda=532 \mathrm{~nm}, \mathrm{FWHM}=$ $10 \mathrm{~ns})$ is used as the laser pulse source in our experiments. 
The setup utilized to detect the time-resolved displacement of the reflecting sample surface consists of a modified Michelson interferometer, as described in Ref. [13]. The setup is the same as previously used by Kneier et al. [14] and described in detail there.

The sample is irradiated with the Nd:YAG pulse on the interferometer side. The time resolution is given by the FWHMs of a test fs laser pulse as 775 ps and 510 ps for the two detectors in the balanced setup, respectively. The vertical displacement resolution is $0.55 \mathrm{~nm}$ for single shots and $0.28 \mathrm{~nm}$ for multi-shot experiments. The probe laser spot focus diameter on the sample is $100 \mu \mathrm{m}$. The Nd:YAG laser pulse irradiates the sample at an angle of incidence of $44^{\circ}$ and is only slightly focussed ensuring a larger spot size than the interferometric probe beam on the sample.

\section{Results and discussion}

\subsection{Reflectivity change}

The interferometric signal is affected by both the vertical displacement of the surface and the change in the reflectivity due to the induced heating from the ns-laser pulse. The utilized two-detector setup determines the reflectivity change from the sum of the inverse signals and takes the result into account in order to determine the surface displacement. Additionally, interpreting the transient reflectivity change yields direct information about the phase state during irradiation. This is crucial for the determination of the times at which phase-shift changes must be accounted for, as will be discussed in Sect. 3.2.

In order to theoretically predict the reflectivity change during thermal heating, the variation of the optical constants with temperature is required. Temperature dependence of $n$ and $k$ for gold has not been given much attention in the literature as for example silicon has received. Otter [15] determined specific data points which are used here to calculate the reflectivity for the layer system of $40 \mathrm{~nm}$ gold on a thin chromium adhesive layer on a silicon substrate at different temperatures. The results are displayed in Fig. 1. In summary, the expected relative reflectivity $(R)$ increase ranges up to $8.5 \%$ before melting of the gold layer. Upon melting, a leap into two-digit magnitude in change is predicted and a theoretical value of $14 \%$ is observed. As an experimental result, Fig. 2 shows time-resolved reflectivity curves for three fluences. At $248 \mathrm{~mJ} / \mathrm{cm}^{2}$ the reffectivity change still remains within the limits expected before melting occurs. In contrast, at $317 \mathrm{~mJ} / \mathrm{cm}^{2}$ the experimentally determined magnitude is $20 \%$, which suggests thorough melting of the $40 \mathrm{~nm}$ gold film at this fluence.

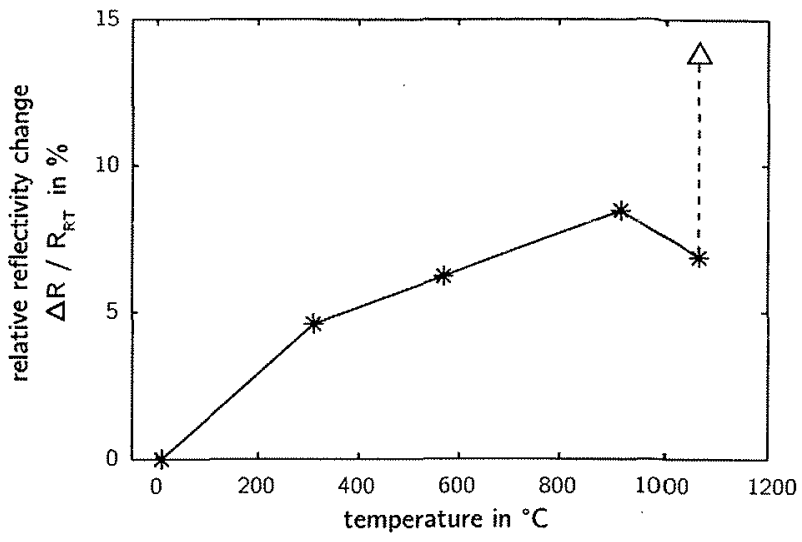

Fig. 1 Temperature dependence of the reflectivity with respect to room temperature for the layer system gold/chromium adhesive layer/silicon substrate. Data points are calculated for $\lambda=488 \mathrm{~nm}$, with reflectivities obtained by interpolation from literature values [15]; * data points represent solid, $\triangle$ points liquid gold

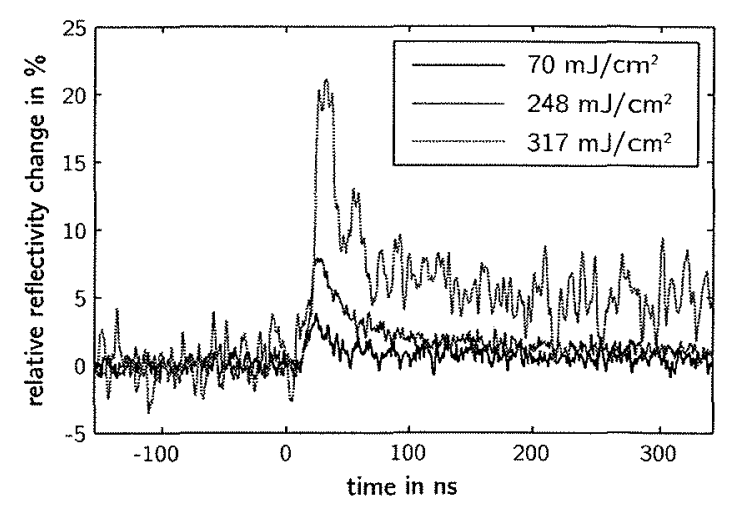

Fig. 2 Transient relative reflectivity change in regime $I$ at three different fluences

\subsection{Phase-shift change}

As has been previously observed for silicon substrate samples [14], the phase shift at the reflection interface of the sample for the interferometer beam changes if part or all of the gold film is melted by the heat of the irradiated ns pulse. The phase of the complex amplitude reflection coefficient at the interface from medium 0 to medium 1

$r=\frac{\hat{n}_{0}-\hat{n}_{1}}{\hat{n}_{0}+\hat{n}_{1}}$

for a metal like gold is, in general, unequal to 0 or $\pi$ (as would be the case for non-conducting materials where the refractive index is to a good approximation purely real). For any fixed phase shift this poses no problem as active stabilization allows us to set the reference point in the interference pattern at the start of the experiment. However, if the gold surface turns liquid during the irradiation, the optical constants $n_{\lambda}$ and $k_{\lambda}$ in the complex refractive index $\hat{n}$ 


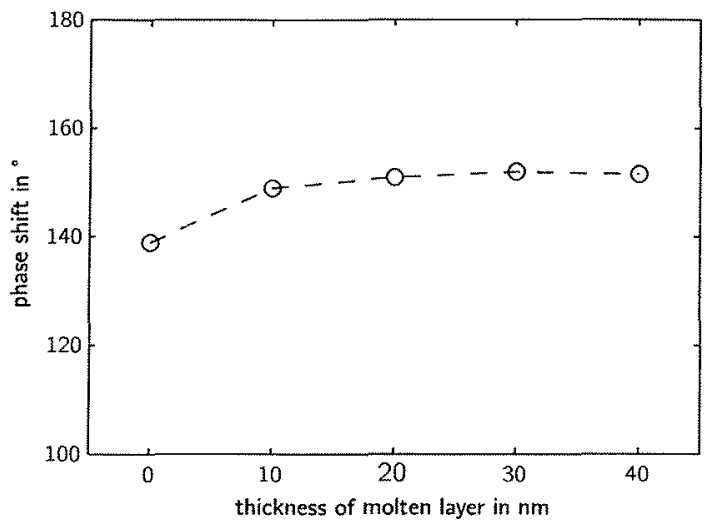

Fig. 3 Phase shift occurring upon reflection of $488 \mathrm{~nm}$ light for the system liquid gold on solid gold background, incident from air. Results are plotted as a function of layer thickness of the molten gold

change. The reflection coefficient for the growing thin film of molten gold on a solid background of the material and the silicon substrate is further complicated by multiple-beam interference. Subsequently, the phase shift upon reflection changes transiently during the experiment. We have here calculated the phase shift by means of the transfer-matrix method as a function of the molten thickness for the wavelength $\lambda=488 \mathrm{~nm}$ using the optical constants for liquid gold from Otter [15]. The results are shown in Fig. 3. For a fully molten gold film, the phase shift changes by approximately $+10^{\circ}$. During the time interval that has to be identified from the transient reflectivity change (see Sect. 3.1), a corrected phase value needs to be taken into account in the determination of the surface displacement from the interferometric signals. For low fluences below the melting threshold this error is irrelevant. For higher fluences a characteristic overshoot appears in the uncorrected displacement just before a plateau is reached, as shown in Fig. 4. Without considering the phase-shift change due to melting, the analysis of the interferometer signal, as shown in Fig. 4(a), yields an overestimation of the displacement by $6.4 \mathrm{~nm}$ when the whole film is molten. Taking the phase-shift change into account, we obtain the corrected displacement with the transition zones being left out in Fig. 4(b). The transition zones of partially melted films are excluded as the exact temporal evolution of intermediate phase shifts is difficult to take into account quantitatively. Disadvantageously, omitting the transition zones prevents correct differentiation (and in particular surface-velocity deduction). In the following analysis, therefore, the erroneous curves are being used for high fluences as well in order to obtain smooth curves for differentiation. Afterwards, the determined maximum values are corrected manually. Within a transition zone, the real values are between the measured (i.e. uncorrected) and the overcorrected values (i.e. fully molten film correction). Veloci-
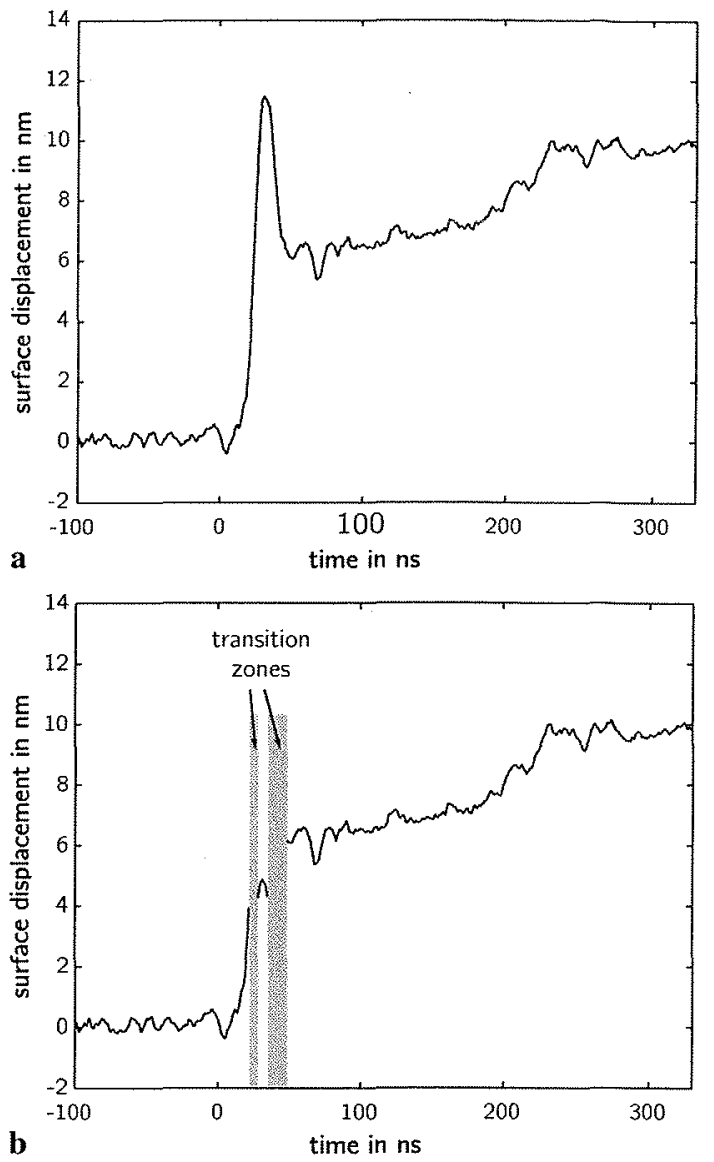

Fig. 4 (a) Surface displacement of a gold film with $\mathrm{Cr}$ layer for $317 \mathrm{~mJ} / \mathrm{cm}^{2}$ without phase-shift correction. For this fluence melting occurs (see Sect. 3.1). (b) Expansion qualitatively corrected in the time span when the reflectivity-change plateau occurs. Transition zones are left out (shaded areas)

ties are adjusted by linear scaling assuming that corrected maximum expansion was attained in the same time period.

\subsection{Fluence-dependent regimes}

Systematic measurements with different irradiated fuences show three distinct regimes. Typical observations for each are illustrated in Fig. 5. They can be characterized as follows: the first regime I at low irradiated fluences is characterized by a relatively small expansion and the expected typical increase in the reflectivity due to the heating of the surface. After irradiation ends and the absorbed heat dissipates, the value of the reflectivity gradually relaxes back to its initial magnitude at room temperature. In Sect. 3.4 we show that the transition to a completely molten gold film also takes place within this regime. Regime I extends to a fluence of about $380 \mathrm{~mJ} / \mathrm{cm}^{2}$. In contrast, in regime II of intermediate fluences, a rise in the reflectivity signal to the value for a completely molten gold film is observed, which 
Fig. 5 Gold film measurements: typical results for the three regimes.

(a) Regime I encompasses low fluences and is characterized by the thermal rise in the transient reflection. (b) A transition regime II of intermediate fuences is identified by a steep drop in the transient reflectivity change. (c) Regime III consists of the subsequent high fuences and is characterized by oscillations. The inset shows the first $100 \mathrm{~ns}$ at higher time resolution
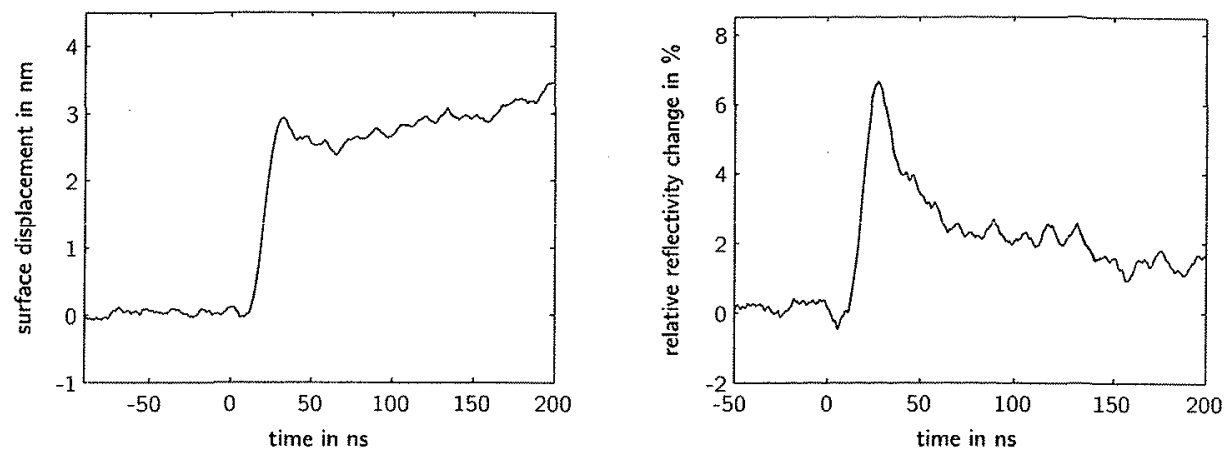
Regime I
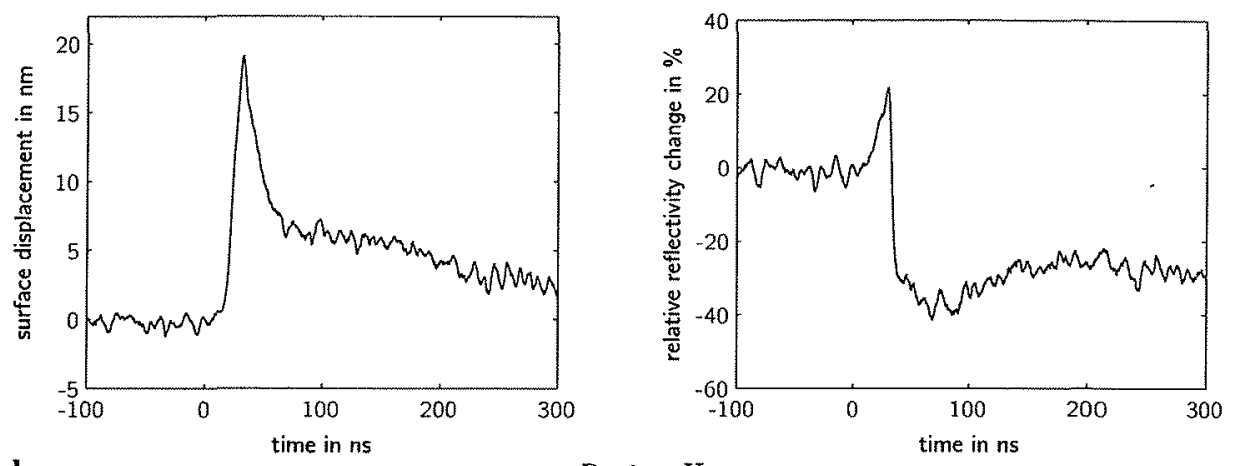

Regime II

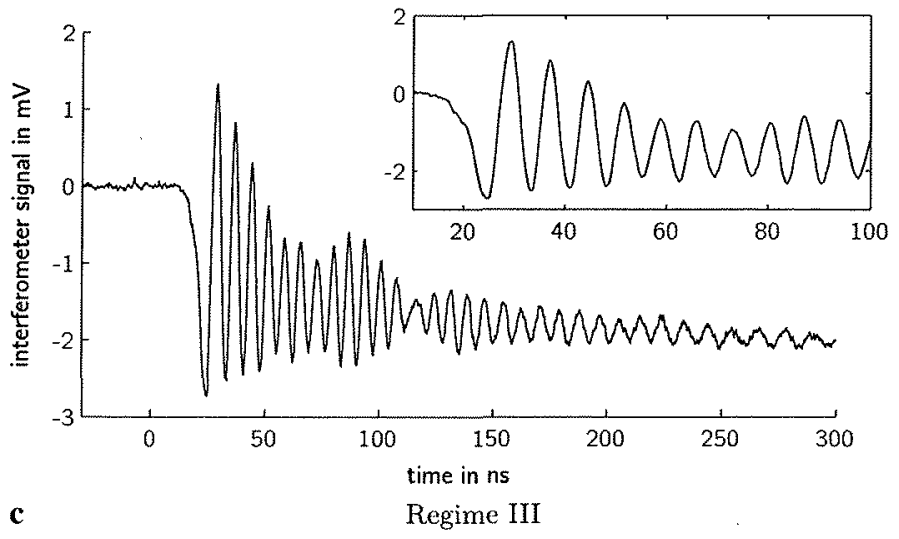

is then followed by a drop (see Fig. 5(b)). The drop increases with larger fluences and reaches a decrease of $60 \%$ from the original magnitude at room temperature at the changeover to regime III. This indicates a dewetting of the liquid gold film as observed by Bischof et al. [16]. This regime ranges to about $470 \mathrm{~mJ} / \mathrm{cm}^{2}$. For even larger fluences in regime III the liquid film lifts off, as will be evidenced in Sect. 3.6. This flying off is registered as oscillations in the interferometer signal. Analysis of the peaks yields the travelled distances and therefore the velocity of the flying film. For the samples with an adhesive chromium layer between film and substrate, this occurs at such high fluences that films disintegrate soon after lift off. If a sample without the adhesive chromium layer is used, the range of regime II is greatly re- duced and detaching films occur at lower fluences. They remain intact for up to $100 \mathrm{~ns}$ such that their velocities could be determined.

\subsection{Regime I: reflectivity change}

The analysis of the transient reflectivity change yields information about the phase state of the gold film during irradiation. The systematic investigation shows that all three fluences which are described in Sect. 3.1 and shown in Fig. 2 are within the range of regime $I$. Following the argument of that section, the $40 \mathrm{~nm}$ gold film is thoroughly molten during pulse irradiation already for a fluence of $317 \mathrm{~mJ} / \mathrm{cm}^{2}$. 


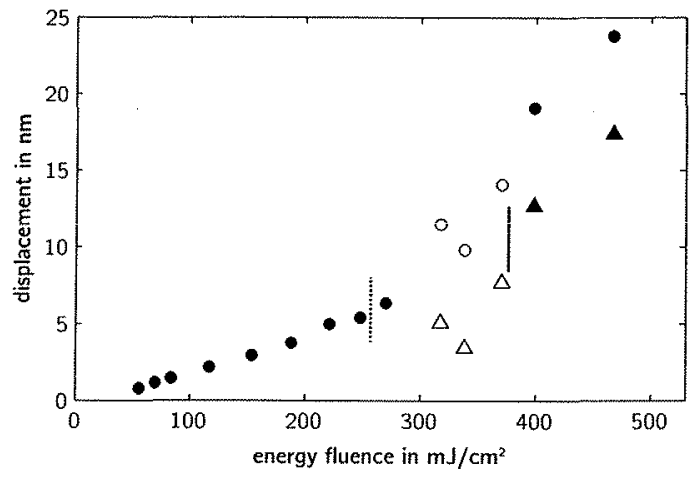

Fig. 6 Maximum displacement of gold film sample. For those fluences at which maximal phase-shift change must be accounted for, corrected values are given by filled triangles. Non-filled data points mark the transition zone with intermediate phase-shift-change magnitude. Real values are expected between $\Delta$ and $O$. Additionally, the transition from regimes I to II is illustrated (solid line) as well as the point at which irradiated pulses begin to permanently affect the surface (dashed line)

Altogether, there is good evidence that the transition to a completely liquid gold film occurs within the first regime.

\subsection{Regimes I and II: thermal expansion}

In order to give a more detailed analysis of the surface dynamics, the maximally achieved displacements are determined in the regimes I and II. The findings are presented in Fig. 6. In addition, the corresponding maximal velocities of the film surface are evaluated. Results are shown in Fig. 7. As discussed in Sect. 3.2, the maximal phase-shift change is accounted for at fluences at which the gold layer is melted completely and displayed in the graph by the filled triangular data points. The transition zone is marked by nonfilled data points. Phase-shift change depends continuously on the thickness of the molten gold layer according to Fig. 3 . Due to the changed thermal expansion in the liquid material, a different slope characteristic is present for fluences with a completely melted gold film compared to lower fluences with solid films throughout the irradiation. The transition between these two gradients in the graphs occurs continuously, with real values expected to be between the uncorrected data $(O)$ and the maximally (i.e. over-) corrected triangular data points $(\triangle)$.

Bischof [17] has calculated for gold films on a glass substrate that the heat diffusion into the substrate is of the same order of magnitude as the amount spent thermally heating the gold. In the case of silicon substrates the heat conductivity is higher $\left(150 \mathrm{~W} / \mathrm{mK}\right.$ at $20^{\circ} \mathrm{C}$ compared to $\left.0.5 \mathrm{~W} / \mathrm{mK}\right)$; therefore, more heat will be transferred from the film into the substrate in the same time. Thorough melting of the $40 \mathrm{~nm}$ film is observed at approximately $300 \mathrm{~mJ} / \mathrm{cm}^{2}$ of irradiated fluence. Analogous to Bischof's calculations, the required energy for reaching the melting temperature of gold,

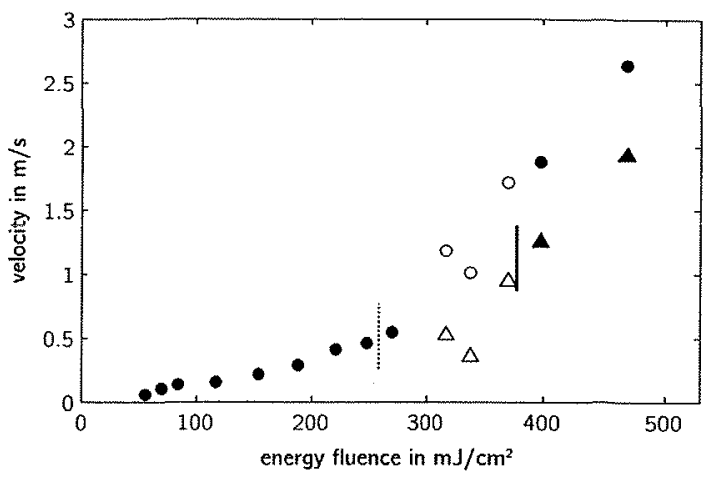

Fig. 7 Maximum surface velocity of gold film sample. For those fluences at which maximal phase-shift change must be accounted for, corrected values are given by filled triangles. Non-filled data points mark the transition zone with intermediate phase-shift-change magnitude. Real values are expected between $\Delta$ and $O$. Additionally, the transition from regimes I to II is illustrated (solid line) as well as the point at which irradiated pulses begin to permanently affect the surface (dashed line)

$T_{\mathrm{m}}=1336 \mathrm{~K}$, in the whole film is $12.35 \mathrm{~mJ} / \mathrm{cm}^{2}$ and the required melting enthalpy is $5.00 \mathrm{~mJ} / \mathrm{cm}^{2}$. The given irradiation setup ( $44^{\circ}$ incidence, $\mathrm{p}$-polarized, $\lambda=532 \mathrm{~nm}$ ) yields reflection $R=50 \%$, transmission $T=20 \%$ and absorption $A=30 \%$. This corresponds to an absorbed fluence of $90 \mathrm{~mJ} / \mathrm{cm}^{2}$ of which approximately $70 \mathrm{~mJ} / \mathrm{cm}^{2}$ is transferred to the substrate. Additionally, the silicon substrate absorbs the transmitted fluence, giving a sum of $130 \mathrm{~mJ} / \mathrm{cm}^{2}$. Measurements of silicon substrates show that silicon expands by about $3 \mathrm{~nm}$ due to this specific heat flow volume [14] and the gold film by $0.7 \mathrm{~nm}$ at $T_{\mathrm{m}}$ according to linear thermal expansion [18]. Measured displacement is, however, on the order of 5-6 nm. A possible explanation could be the frustrated expansion from the borders of the heated area bounded by unheated material, which gives rise to further expansion in the $z$-direction. Considering the volume expansion coefficient instead yields an expansion of the gold film at $T_{\mathrm{m}}$ of $2.1 \mathrm{~nm}$, and, therefore, substrate and film expansion together then agree well with the determined displacement.

\subsection{Regime III: film detachment}

The occurrence of oscillations in the interferometric signal suggests that the deposited energy quantity is sufficient to lift off the gold films and see them fly off from the substrate. The film flight velocities can be evaluated from the interferometric signal. The results for two irradiation fluences are shown in Fig. 8. A first phase of acceleration can be observed, followed by a short plateau of relatively constant velocity. After about $60 \mathrm{~ns}$, a slow continuous decline in the velocity becomes apparent. Peak heights in the interferometric signal decrease with time. The observed film velocities are of the order of $20-40 \mathrm{~m} / \mathrm{s}$. Two possible scenarios for 


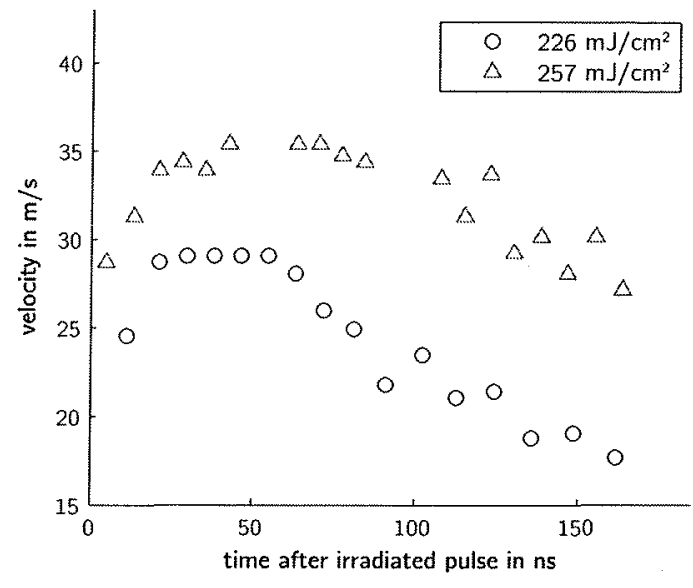

Fig. 8 Velocities of the flying gold films

the process come to mind: (i) either droplets form on the surface due to the intense laser irradiation which leave the surface as an ensemble of various sizes or (ii) the liquid film lifts off as a whole. In the case of the first model, droplets would form in the gold surface through dewetting. These would leave the surface analogous to the gold nanotriangles in the experiments by Habenicht et al. [19]. The velocity of the droplets arises from surface minimization and would therefore be size dependent. Due to the grain-size distribution in the evaporated film, one would expect a droplet-size distribution which would lead to a large distribution of different velocities, as opposed to the observed single velocity. For this reason we favor the second model. According to it, the gold film experiences upward expansion. Thereby, the center of mass is accelerated upwards as well. If the upwarddirected force exceeds the adhesive forces to the substrate, the film lifts off and flies away as a whole. Simultaneously, the characteristic dewetting holes and spinodals are beginning to form in the liquid film. Before this instability can occur, however, the film is already airborne. With time the film disintegrates due to progressive droplet formation on the basis of the inchoated dewetting holes. Therefore, the oscillating signal in the interferometer diminishes over time. This is further supported by the occurrence of a single velocity which does not remain constant.

Additionally, experiments with flying liquid films [20, 21] predict that deceleration is dependent on the total mass of the flying film. In order to investigate this specific behavior, samples of thin tantalum films have been examined. These samples showed the same characteristic behavior of the three energy regimes. The third consisted of the flying thin tantalum films with the advantage that two samples were available with $50 \mathrm{~nm}$ and $100 \mathrm{~nm}$ film thickness, respectively. A comparison of the velocities between the two films is given in Fig. 9. The $100 \mathrm{~nm}$ film has twice the mass of the $50 \mathrm{~nm}$ film. At the same time the deceleration takes place

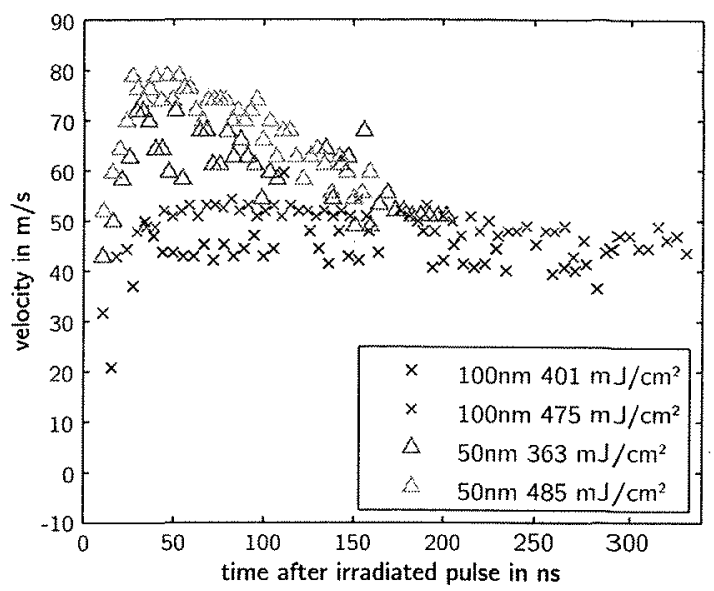

Fig. 9 Velocity of the flying tantalum films at two representative fluences per sample

notably more slowly, an observation intuitively explainable by the larger mass that is slowed by the same number of air molecules and, therefore, agreeing with the prediction. Although these explanations fit the experimental findings, one aspect remains remarkable. The velocity of the detaching films of $30 \mathrm{~m} / \mathrm{s}$ is surprisingly high compared to the velocities which are measured in regimes I and II. One might argue that an additional acceleration process contributes, which could be the evaporation of a layer formed at the interface to the substrate at the high-vacuum evaporation condition used.

Finally, the periodic constrictions in the oscillatory signal in Fig. 5(c) can be interpreted as the formation of vibrations of the flying liquid membrane. The periodicity of the envelope is of the order of $10 \mathrm{MHz}$. Observations for crystalline membranes generally confirm the existence of oscillations with frequencies of this order of magnitude. The lack of comparative experiments for free liquid membranes leads to the necessity to conduct further research to conclusively explain this observed feature.

\section{Conclusion}

In conclusion, our experiments show the nanomechanical surface displacement of a $40 \mathrm{~nm}$ gold film on a silicon substrate on the nanosecond time scale. The presence of three distinct energy regimes is observed. They can be characterized by the behavior of the transient reflectivity, dewetting processes and lift off of whole liquid films. The film surface dynamics is observed at and above the melting threshold of gold by integrating the occurrence of a phase-shift change during the irradiation in these instances. The transition from solid to molten gold films is observed by means of the maximum surface displacement and the maximum velocities. The 
transition from solid to a completely molten gold film in the observed maximum displacement height is only in good quantitative agreement with the expected density change at the melting point if the phase-shift change is taken into account. Including phase-shift change yields a difference of approximately $4 \mathrm{~nm}$ equating to the $10 \%$ change in density of the $40 \mathrm{~nm}$ gold film at the melting point. The velocities of the film surface are determined to be $0.6 \mathrm{~m} / \mathrm{s}$ and $1.9 \mathrm{~m} / \mathrm{s}$ below and above the melting threshold, respectively.

Further phenomena include the occurrence of dewetting and, at even higher irradiated laser fluences, the flying off of liquid films. Systematic measurements evidence that thin films detach as a whole and fly intact for many nanoseconds with velocities of $20-40 \mathrm{~m} / \mathrm{s}(30-70 \mathrm{~m} / \mathrm{s}$ for tantalum films) before disintegrating into droplets. The values obtained for the surface displacement on the nanosecond scale through irradiation with ns-laser pulses provide basic knowledge necessary in many nanomechanical applications like laser cleaning or the creation of nanostructures, and especially concerning the latter's method of using thin films of materials which expand upon melting.

Acknowledgements We gratefully acknowledge the financial support of the Center of Applied Photonics (CAP) of the University of Konstanz as well as the SFB 767 of the DFG and the Strategic Japanese-German Cooperative Program of the JST and DFG.

\section{References}

1. M. Schmotz, D. Gollmer, F. Habel, S. Riedel, P. Leiderer, Chin. J. Phys. 49, 527 (2011)

2. P. Voigt, R. Koch, J. Appl. Phys. 92, 7160 (2002)
3. J. Jersch, F. Demming, I. Fedotov, K. Dickmann, Rev, Sci. Instrum. 70, 4579 (1999)

4. T. Hesjedal, E. Chilla, H.-J. Fröhlich, Appl. Phys. Lett. 69, 354 (1996)

5. P. Chen, I.V. Tomov, P.M. Rentzepis, Appl. Phys. Lett. 71, 1646 (1997)

6. B. Lings, J.S. Wark, M.F. DeCamp, D.A. Reis, S. Fahy, J. Phys. Condens. Matter 18, 9231 (2006)

7. M.F. DeCamp, D.A. Reis, A. Cavalieri, P.H. Bucksbaum, R. Clarke, R. Merlin, E.M. Dufresne, D.A. Arms, A.M. Lindenberg, A.G. MacPhee, Z. Chang, B. Lings, J.S. Wark, S. Fahy, Phys. Rev. Lett. 91, 165502 (2003)

8. V. Dobler, R. Oltra, J.P. Boquillon, M. Mosbacher, J. Boneberg, P. Leiderer, Appl. Phys. A 69, 335 (1999)

9. B. Audoin, H. Meri, C. Rossignol, Phys. Rev. B 74, 214304 (2006)

10. T. Tachizaki, T. Muroya, O. Matsuda, Y. Sugawara, D.H. Hurley, O.B. Wright, Rev. Sci. Instrum. 77, 043713 (2006)

11. T. Geldhauser, F. Ziese, F. Merkt, A. Erbe, J. Boneberg, P. Leiderer, Appl. Phys. A 89, 109 (2007)

12. S. Riedel, M. Schmotz, P. Leiderer, J. Boneberg, Appl. Phys. A 101, $309(2010)$

13. C.B. Scruby, L.E. Drain, Laser Ultrasonics-Techniques and Applications (Adam Hilger, Bristol, 1990)

14. F. Kneier, T. Geldhauser, P. Leiderer, J. Boneberg, Appl. Phys. A $105,25(2011)$

15. M. Otter, Z. Phys. A - Hadrons Nucl. 161, 539 (1961)

16. J. Bischof, D. Scherer, S. Herminghaus, P. Leiderer, Phys. Rev, Lett. 77, 1536 (1996)

17. J. Bischof, Ph.D. thesis, UFO-Dissertationen 313 , UFO Atelier für Gestaltung und Verlag, Allensbach, ISBN 3-930803-12-7 (1997)

18. Y.S. Touloukian, R.K. Kirby, E.R. Taylor, T.Y.R. Lee, Thermal Expansion: Metallic Elements and Alloys. Thermophysical Properties of Matter, vol. 12 (IFl/Plenum, New York, 1975)

19. A. Habenicht, M. Olapinski, F. Burmeister, P. Leiderer, J. Boneberg, Science 309, 2043 (2005)

20. F. Lang, P. Leiderer, S. Georgiou, Appl. Phys. Lett. 85, 2759 (2004)

21. P. Frank, J. Graf, F. Lang, J. Boneberg, P. Leiderer, Appl. Phys. A 101, $7(2010)$ 